\title{
Policultivo do camarão de água doce Macrobrachium amazonicum (Heller, 1862) com a Tilápia do Nilo (Oreochromis niloticus) alimentadas com rações peletizada e farelada
}

\section{Polyculture of fresh water shrimp Macrobrachium amazonicum (Heller, 1862) wifh Nile tilapia (Oreochromis niloticus) feeding with ration pelleted and mashed}

\author{
Bruno Estevão de Souza ${ }^{1 *}$; Leonardo Luiz Stringuetta ${ }^{2}$; Adriana Cristina \\ Bordignon $^{2}$; Leandro Bohnenberger ${ }^{2}$; Wilson Rogério Boscolo ${ }^{3}$; Aldi Feiden ${ }^{3}$
}

\section{Resumo}

O objetivo deste trabalho foi avaliar a influência do camarão de água doce Macrobrachium amazonicum (Heller, 1862) no desempenho da Tilápia do Nilo (Oreochromis niloticus) cultivada no sistema de policultivo e alimentada com rações peletizadas e fareladas. $\mathrm{O}$ trabalho foi realizado no Centro de Pesquisa em Aqüicultura Ambiental-CPAA/IAP - Toledo/PR por um período de 37 dias. Utilizaram-se como unidades experimentais 16 tanques escavados, revestidos com concreto e com fundo de terra, com dimensão de $4 \times 3 \mathrm{~m}$ e volume útil de $3,5 \mathrm{~m}^{3}$. Foram utilizadas 30 tilápias e 150 camarões por unidade experimental distribuídos em um delineamento inteiramente ao acaso com 4 tratamentos e 4 repetições, sendo TF: tilápia alimentadas com ração farelada; TCF: tilápia e camarão alimentados com ração farelada; TP: tilápia alimentada com ração peletizada e TCP: tilápia e camarão alimentados com ração peletizada. A densidade utilizada foi de 2,6 peixes $/ \mathrm{m}^{2}$ com peso inicial médio de $5,58 \pm 0,10 \mathrm{~g}$ e comprimento inicial médio de 5,56 cm, sendo a densidade de camarões de 13 camarões $/ \mathrm{m}^{2}$ com comprimento inicial médio de $1,04 \mathrm{~cm}$. A temperatura foi aferida diariamente, enquanto que as variáveis oxigênio dissolvido, $\mathrm{pH}$ e condutividade elétrica, semanalmente. A quantidade de ração fornecida foi de $10 \%$ da biomassa total dos peixes, com freqüência alimentar de 4 vezes ao dia, sendo corrigida semanalmente em função das biometrias. Durante o período experimental os valores médios de temperatura, oxigênio dissolvido, $\mathrm{pH}$ e condutividade elétrica da água dos tanques foram de $23,42 \pm 0,83{ }^{\circ} \mathrm{C}, 5,32 \pm 0,52 \mathrm{mg} / \mathrm{L}, 7,02 \pm 0,39$, e $1,00 \pm 0,01 \mathrm{mS} / \mathrm{cm}$ respectivamente. Não se registrando qualquer influência do camarão durante o cultivo e a ração peletizada proporcionou uma melhor conversão alimentar e desempenho das tilápias. Palavras-chave: Processamento, ração, desempenho produtivo, peixe.

\begin{abstract}
The objective of this work was to evaluate the influence of fresh water shrimp Macrobrachium amazonicum (Heller, 1862) in performance of Nile tilapia (Oreochromis niloticus) cultivated in polyculture system and feeding with ration pelleted and mashed. The work was realized in Centro de Pesquisa em Aqüicultura Ambiental-CPAA/IAP - Toledo/PR during 37 days. Were utilized like experimental unit 16 ponds excavated, covered with concrete but with bottom of soil with dimension the 4 x $3 \mathrm{~m}$ and useful volume the $3,5 \mathrm{~m}^{3}$. Were utilized 30 tilapias e 150 shrimps for experimental unit distributed at an entirely
\end{abstract}

1 Engenheiro de Pesca, Doutorando do Centro de Aqüicultura da UNESP - CAUNESP, Jaboticabal-SP. E-mail: bruno_bes@ yahoo.com.br

2 Engenheiro de Pesca.

3 Dr. Professor Titular do Curso de Engenharia de Pesca da Universidade Estadual do Oeste do Paraná - UNIOESTE, Toledo-PR.

* Autor para correspondência 
randomized design with 4 treatments and 4 replications, where TF: tilapia feeding with ration mashed; TCF: tilapia and shrimp feeding with ration mashed; TP: tilapia feeding with ration pelleted; TCP: tilapia and shrimp feeding with ration pelleted. The density used were the $2,6 \mathrm{fishes} / \mathrm{m}^{2}$ with medium initial weight the $5,58 \pm 0,10 \mathrm{~g}$ and initial length the $5,56 \mathrm{~cm}$, and the density of shrimp was the $13 \mathrm{shrimps} / \mathrm{m}^{2}$ with initial length the $1,04 \mathrm{~cm}$. The temperature was gauged daily, while the variables dissolved oxygen, $\mathrm{pH}$ and electrical conductivity, weekly. The quantity of ration supplied was the $10 \%$ of total biomass of fishes, with feed frequency the 4 times a day, being corrected weekly in function of the biometry. During the experimental period the medium values of temperature, dissolved oxygen, $\mathrm{pH}$ and electrical conductivity of the ponds water were $23,42 \pm 0,83^{\circ} \mathrm{C}, 5,32 \pm 0,52 \mathrm{mg} / \mathrm{L}, 7,02 \pm 0,39$, e $100,96 \pm 1,81 \mu \mathrm{S} /$ $\mathrm{cm}$ respectively. Won't registering any influence of shrimp during the cultivation and the ration pelleted provide the better conversion alimentary and performance of tilapias.

Key words: Diet processing, productive performance, fish

\section{Introdução}

Segundo dados da Food and Agriculture Organization - FAO (2008), no ano de 2006 foram produzidas 162,2 milhões de toneladas de pescado provenientes da aqüicultura e pesca. A aqüicultura contribuiu com mais de 66,7 milhões de toneladas, apresentando um crescimento de 5,2 \% em relação ao ano anterior (2005). Considerando este perfil, entre as diversas espécies de peixes exóticas cultivadas no país, a tilápia (Oreochromis niloticus), representou um total de produção de 32.460 toneladas no ano de 2000, o que corresponde a 30,9 $\%$ do total produzido pelo setor aqüícola brasileiro (BOSCARDIN-BORGHETTI; OSTRENSKY; BORGHETTI, 2003).

A tilápia do Nilo (O. niloticus) é uma espécie bastante rústica, de hábito alimentar fitoplanctófago, que aceita também alimentos artificiais. Apresenta carne de excelente sabor e qualidade e o filé, principal produto de sua industrialização, possui ótima aceitação no mercado consumidor, além de ser muito apreciada em pesque-pagues para a pesca esportiva. Estes fatores tornam a espécie de grande interesse para a piscicultura (BOSCOLO, 2003).

A produção de camarões de água doce do gênero Macrobrachium vem crescendo muito, com a produção mundial tendo atingido 300.000 toneladas no ano de 2001 (VALENTI, 2004). O camarão Macrobrachium amazonicum (HELLER, 1862) é a espécie da família Palaemonidae de água doce mais comumente encontrada no Brasil (LIMA; ODINETZ
COLLART, 1997), ocorrendo em lagos e rios da Amazônia Central (CHAVES; MAGALHÃES, 1993). Esta espécie tem sido introduzida em grandes açudes públicos e reservatórios para servir de alimento para peixes carnívoros e recurso pesqueiro para as populações (TORLONI et al., 1993). Devido ao rápido crescimento e fácil manutenção em cativeiro o Macrobrachium amazonicum tem despertado um interesse crescente para o cultivo comercial (LIRA; SILVA; CHAVES, 2003).

O policultivo é o ato de criar diferentes espécies em conjunto, num mesmo viveiro, quando mais de uma delas tem interesse para o produtor (ZIMMERMANN, 1991). Hepher e Pruginin (1981) afirmam que o aspecto mais importante do policultivo é o aumento da produtividade pela melhor utilização do alimento natural.

No policultivo de tilápias e camarões de água doce o manejo alimentar normalmente é realizado enfocando os peixes, pois os camarões conseguem aproveitar muito bem os restos alimentares, as fezes e os nutrientes depositados no fundo dos viveiros (SANTOS, 2001). Para os produtores de peixes, a colocação de camarões em baixa densidade produz uma considerável receita adicional, devido ao alto valor de mercado dos crustáceos (ZIMMERMANN, 1991).

Neste sentido, o presente trabalho tem como objetivo avaliar a influência do camarão de água doce $M$. amazonicum no desempenho da Tilápia do Nilo (O. niloticus) alimentados com rações peletizadas e fareladas. 


\section{Material e Métodos}

O trabalho foi realizado no Centro de Pesquisa em Aqüicultura Ambiental/IAP - Toledo/PR por um período de 37 dias. Os alevinos de tilápia utilizados foram provenientes de estações comerciais de cultivos e os camarões utilizados foram coletados com rede de arrasto no reservatório de Itaipu no município de Entre Rios do Oeste - PR. Utilizaramse como unidades experimentais 16 tanques escavados, revestidos com concreto, com fundo de terra de dimensões de $4 \times 3 \mathrm{~m}$, volume útil de 3,5 $\mathrm{m}^{3}$ e renovação de água apenas para compensar a água evaporada e infiltrada. Foram estabelecidos 4 tratamentos com 4 repetições, num delineamento inteiramente ao acaso: TF: tilápia alimentadas com ração farelada; TCF: tilápia e camarão alimentados com ração farelada; TP: tilápia alimentada com ração peletizada e TCP: tilápia e camarão alimentados com ração peletizada.

A densidade de estocagem utilizada foi de 2,6 peixes $/ \mathrm{m}^{2}$ com peso inicial médio de $5,58 \pm 0,10$ g e comprimento inicial médio de $5,56 \mathrm{~cm}$, sendo a densidade de camarões de 13 camarões $/ \mathrm{m}^{2}$ com comprimento inicial médio de $1,04 \mathrm{~cm}$. Os camarões foram soltos uma semana antes das tilápias.

O arraçoamento foi de $10 \%$ da biomassa total dos peixes, com freqüência alimentar de quatro vezes ao dia, duas no período da manhã e duas no período da tarde (8:00, 11:00, 15:00 e 18:00hs), sendo corrigido semanalmente em função das biometrias. As rações utilizadas na forma farelada e peletizada apresentavam a mesma composição percentual e química, contendo $33 \%$ de proteína bruta e 3100 Kcal de energia digestível por $\mathrm{kg}$ de ração. As rações foram formuladas à base de farelo de soja, farinha de peixe e milho (Tabela 1). Os alimentos foram moídos em triturador tipo faca com peneira de $0,5 \mathrm{~mm}$. Para a peletização a ração foi umedecida com água a $55^{\circ} \mathrm{C}$ e peletizada em um moinho de carne elétrico. Posteriormente as rações foram secas em estufa de ventilação forçada por 24 horas.

Tabela 1. Composição percentual e química das rações utilizadas na alimentação das tilápias e camarões.

\begin{tabular}{lc}
\hline Ingredientes & $\%$ \\
\hline Milho & 33,80 \\
Farelo de soja & 51,76 \\
Farinha de tilápias & 12,92 \\
Sal comum & 0,50 \\
Suplemento min. + vit. $^{1}$ & 1,00 \\
Antioxidante (BHT) & 0,02 \\
\hline Total & 100,00 \\
\hline Composição & $\%$ \\
\hline Energia digestível(kcal/kg) & 3100 \\
Proteína bruta & 33,57 \\
Proteína digestível & 30,00 \\
Gordura & 4,70 \\
Fibra total & 3,71 \\
Lisina & 1,92 \\
Metionina & 0,54 \\
Metionina+cistina & 1,18 \\
Amido & 28,05 \\
Cálcio & 1,15 \\
Fósforo total & 0,75 \\
\hline
\end{tabular}

${ }^{1}$ Níveis de garantia por quilograma do produto: Vit. A, 500.000UI; Vit. D, 200.000UI; Vit. E, 5.000mg; Vit. K3, 1.000mg; Vit. B1, 1.500mg; Vit. B2, 1.500mg; Vit. B6, 1.500mg; Vit. B12, 4.000mg; Ác. Fólico, 500mg; Pantotenato Ca, 4.000mg; Vit. C, 15.000mg; Biotina, 50mg; Inositol, 10.000; Nicotinamida, 7.000; Colina, 40.000mg; Co, 10mg; Cu, 500mg; Fe, 5.000mg; I, 50mg; Mn, $1500 \mathrm{mg}$; Se, 10mg; Zn, $5.000 \mathrm{mg}$. 
Ao final do período experimental as tilápias e camarões foram pesados e medidos para avaliação do peso final médio, comprimento final médio, sobrevivência e conversão alimentar aparente. Os dados obtidos foram submetidas à análise estatística (análise de variância - ANOVA) pelo programa estatístico SAEG (Sistema de Análises Estatísticas e Genética) (UNIVERSIDADE FEDERAL DE VIÇOSA - UFV, 1997).

\section{Resultados e Discussão}

Durante o período experimental os valores médios de temperatura, oxigênio dissolvido, $\mathrm{pH}$ e condutividade elétrica da água dos tanques foram de $23,42 \pm 0,83{ }^{\circ} \mathrm{C}, 5,32 \pm 0,52 \mathrm{mg} / \mathrm{L}, 7,02 \pm 0,39$, e $1,00 \pm 0,01 \mathrm{mS} / \mathrm{cm}$ respectivamente permanecendo dentro dos níveis aceitáveis para a aqüicultura (BOYD, 1990).

Os resultados referentes ao desempenho dos alevinos de tilápia do Nilo criadas em sistema de policultivo com o camarão $M$. amazonicum estão demonstrados na Tabela 2.

Tabela 2. Valores médios de desempenho dos alevinos de tilápia do Nilo criadas em sistema de policultivo com camarão Macrobrachium amazonicum.

\begin{tabular}{lccccc}
\hline \multirow{2}{*}{ Parâmetros } & \multicolumn{4}{c}{ Tratamentos } & \multirow{2}{*}{$\mathrm{CV}(\%)$} \\
\cline { 2 - 5 } & $\mathrm{TF}^{1}$ & $\mathrm{TCF}^{2}$ & $\mathrm{TP}^{3}$ & $\mathrm{TCP}^{4}$ & \\
\hline Peso inicial (g) & $5,53^{\mathrm{a}}$ & $5,58^{\mathrm{a}}$ & $5,57^{\mathrm{a}}$ & $5,65^{\mathrm{a}}$ & $1,78^{\mathrm{NS}}$ \\
Peso final (g) & $12,89^{\mathrm{ab}}$ & $12,00^{\mathrm{b}}$ & $14,20^{\mathrm{a}}$ & $14,13^{\mathrm{a}}$ & $6,27^{*}$ \\
Comprimento final (cm) & $7,37^{\mathrm{b}}$ & $7,17^{\mathrm{c}}$ & $7,50^{\mathrm{ab}}$ & $7,55^{\mathrm{a}}$ & $1,36^{*}$ \\
Conversão alimentar aparente & $2,03^{\mathrm{ab}}$ & $2,37^{\mathrm{a}}$ & $1,75^{\mathrm{b}}$ & $1,76^{\mathrm{b}}$ & $11,53^{*}$ \\
Sobrevivência (\%) & $88,16^{\mathrm{a}}$ & $88,33^{\mathrm{a}}$ & $88,33^{\mathrm{a}}$ & $85,33^{\mathrm{a}}$ & $7,70^{\mathrm{NS}}$ \\
\hline
\end{tabular}

* Médias na mesma linha seguidas de letras distintas, diferem $(\mathrm{p}<0,05)$ pelo teste de Duncan.

$\mathrm{TF}^{1}$ - Tilápia tratada com ração farelada;

$\mathrm{TCF}^{2}$ - Tilápia e camarão tratados com ração farelada;

$\mathrm{TP}^{3}$ - Tilápia tratada com ração peletizada;

$\mathrm{TCP}^{4}$ - Tilápia e camarão tratados com ração peletizada.

Para as variáveis peso final, comprimento final e conversão alimentar das tilápias foram observadas diferenças $(\mathrm{P}<0,05)$ entre os diferentes tratamentos. No entanto, a sobrevivência das tilápias não foi afetada pelo policultivo com o camarão e processamento da ração.

$\mathrm{O}$ peso final apresentou diferenças $(\mathrm{P}<0,05)$ entre os tratamentos, com melhores resultados observados no tratamento $\mathrm{TP}$, não diferindo de TCP e TF. O pior resultado $(\mathrm{P}<0,05)$ foi observado no tratamento TCF, mas não divergiu $(\mathrm{P}>0,05)$ do tratamento TF. Estes resultados indicam que o peso final das tilápias não foi influenciado pela presença dos camarões no tanque.
Em relação ao comprimento final houve diferenças $(\mathrm{P}<0,05)$ entre os tratamentos, sendo que o maior valor foi observado nas tilápias do tratamento TCP, não diferindo dos animais do tratamento TP. $\mathrm{O}$ pior comprimento final médio foi observado no tratamento TCF. Resultados intermediários de comprimento final médio foram observados para o tratamento TF, não diferindo do tratamento TP.

Quanto a conversão alimentar, os dados demonstram que os melhores índices foram proporcionados pelo tratamento $\mathrm{TP}$, não diferindo $(\mathrm{P}>0,05)$ do TCP e TF. O pior resultado de conversão alimentar foi observado nas tilápias do tratamento TCF sem apresentar diferença com o tratamento TF. 
Quanto a taxa de sobrevivência das tilápias, não foram observadas diferenças significativas $(\mathrm{P}>0,05)$ entre os diferentes tratamentos. Portanto, nem o policultivo com o camarão nem o processamento da ração inteferiu na sobrevivência das tilápias.
Os valores médios de desempenhoe sobrevivência dos camarões Macrobrachium amazonicum estão apresentados na Tabela 3.

Tabela 3. Valores médios de desempenho e sobrevivência do camarão Macrobrachium amazonicum (HELLER, 1862).

\begin{tabular}{lccc}
\hline \multirow{2}{*}{ Variáveis } & \multicolumn{2}{c}{ Tratamentos } & \multirow{2}{*}{ CV $(\%)$} \\
\cline { 2 - 3 } & TCF & TCP & \\
\hline Comprimento inicial médio $(\mathrm{cm})$ & 1,04 & 1,04 & --- \\
Comprimento final médio $(\mathrm{cm})$ & 2,03 & 2,14 & $5,32^{\mathrm{NS}}$ \\
Sobrevivência $(\%)$ & 71,50 & 77,00 & $8,05^{\mathrm{NS}}$ \\
\hline
\end{tabular}

Os valores médios de comprimento final e sobrevivência para os camarões cultivados em policultivo com a tilápia do Nilo e alimentados com rações fareladas e peletizadas não apresentaram diferenças $(\mathrm{P}>0,05)$.

Segundo Hossain e Islam (2006), o policultivo do gênero Macrobrachium com outras espécies como as carpas demonstraram que os camarões e os peixes podem utilizar diferentes nichos alimentares de maneira eficiente. Engle (1987), que também estudou o policultivo do gênero Macrobrachium com carpas obteve resultados onde o policultivo se mostrou mais lucrativo do que o monocultivo.

Observando os resultados fica nítido que a ração farelada proporcionou em todos os tratamentos o pior desempenho da tilápia, estando de acordo com (DUPREE, 1984), que relatou em seu trabalho que rações fareladas estão sujeitas a grandes perdas dos seus nutrientes, devido a elevada relação entre a sua área superficial. Este fato se deve principalmente há uma maior lixiviação dos nutrientes. Ainda a peletização permite uma maior uniformidade dos ingredientes da ração e uma maior aceitação dos ingredientes da mistura e diminuição da seletividade alimentar pelos peixes.
Avaliando o desempenho de tilápias na fase de crescimento alimentadas com rações fareladas e rações submetidas aos processos de peletização e extrusão, Furuya, Furuya e Souza (1997) também observaram melhor índice de conversão alimentar na ração peletizada.

Os resultados obtidos assemelham-se aos dados encontrados por Baccarin e Camargo (2005), que utilizou diferentes tipos de processamento de ração: peletizada, farelada e extrusada, obtendo um melhor rendimento na ração peletizada.

Segundo Kubitza (1997), no caso de utilização de rações fareladas na alimentação de organismos aquáticos, estas necessitam de suplementação vitamínica e mineral de três a cinco vezes maiores que os níveis de suplementação recomendados para rações granuladas.

Como no presente trabalho as rações apresentavam a mesma composição percentual e química, inclusive com o mesmo nível de suplementação mineral e vitamínica, tanto na ração farelada como peletizada a forma de processamento realmente pode ter interferido no desempenho das tilápias. 
Resultados experimentais aplicados posteriormente como metodologia na produção comercial têm demonstrado a viabilidade técnica e econômica do policultivo da tilápia do Nilo com camarão de água doce conforme citou Santos e Valenti (2002). Pérez e Alston (2000) também recomendam o sistema de policultivo entre a tilápia e o camarão do gênero Macrobrachium para reduzir os custos com a alimentação, além de ser uma alternativa para criadores que utilizam sistema extensivo de monocultivo, sem aeração, e onde o peixe é a colheita principal.

Neste trabalho é possível fazer uma simulação para avaliar economicamente os tratamentos. Pois os tratamentos TF, TP e TCP não foram significativos, o que indica que em termos estatísticos a escolha de um deles é eficiente.

\section{Conclusão}

O Camarão de Água Doce Macrobrachium amazonicum (HELLER, 1862) não influenciou no desempenho da Tilápia do Nilo (Oreochromis niloticus), podendo ser cultivado no sistema de policultivo com essa espécie. Recomenda-se para alevinos de tilápia do Nilo na fase inicial a utilização de rações peletizadas por se manter por mais tempo na água sem desagregar.

\section{Referências}

BACCARIN,A.E.; CAMARGO,A.F.M.Characterization and evaluation of the impact of feed management on the effluents of Nile Tilapia (Oreochromisniloticus). Culture Brazilian Archives of Biology and Technology, Curitiba, v. 48, n. 1, p. 81-90, jan. 2005.

BOSCARDIN-BORGHETTI, N. R.; OSTRENSKY, A.; BORGHETTI, J. R. Aqüicultura: uma visão geral sobre a produção de organismos aquáticos no Brasil e no mundo. Curitiba: Grupo Integrado de Aqüicultura e estudos ambientais, 2003.

BOSCOLO, W. R. Resíduos da industria de filetagem de Tilápias na alimentação da Tilápia do Nilo (Oreochromis niloticus L.). 2003. Tese. (Doutorado em Zootecnia) Universidade Estadual de Maringá, Maringá.
BOYD, C. Water quality in ponds for aquaculture. London: Birmingham, 1990.

CHAVES, P. T.C.; MAGALHÃES, C. O desenvolvimento ovocitário em Macrobrachium amazonicum (Heller, 1862) (Crustácea: Decápoda: Palaemonidae), camarão dulcícola da região amazônica. Acta Amazônica, Manaus, v. 23, n. 1, p. 17-23, 1993.

DUPREE, H. K Feeding practices. In: ROBINSON, E. N., LOVELL. R T. Nutrition and feeding of channell catfish. Auburn: Southem Cooperative, 1984. p. 51-54.

ENGLE, C. R. Analysis economico dela production commercial de la Tilapia. Colossoma y Macrobrachium rosenbergii en mono y policultivo en panama. Revista Latinoamerica Acuiculture, Lima, v. 33, n. 1, p. 6-25, 1987.

FOOD AND AGRICULTURE ORGANIZATION -FAO. Global Aquaculture Production. Disponível em: <http:// www.fao.org/fishery/statistics/global-aquacultureproduction/en>. Acesso em: 4 jun. 2008.

FURUYA, W. M.; FURUYA, V. B.; SOUZA, S. R. Desempenho de tilápias do Nilo (Oreochromis niloticus L.), submetidas a dietas farelada, peletizada e extrusada, na terminação. In: REUNIÃO DA SOCIEDADE BRASILEIRA DE ZOOTECNIA, 34., 1997, Juiz de Fora. Anais... Juiz de Fora: SBZ, 1997. p. 230-231.

HEPHER, R.; PRUGININ, Y. Commercial Fish Farming: with special reference to fish culture is Israel. Nova Iorque: John Wiley, 1981.

HOSSAIN, M. A.; ISLAM, M. S. Optimization of stocking density of freshwater prawn Macrobrachium rosenbergii (de Man) in carp polyculture in Bangladesh. Aquaculture Research, Oxford, v. 37, n. 10, p. 994-1000, 2006.

KUBITZA, F. Nutrição e alimentação dos peixes. Piracicaba: Ed. da Escola Superior de Agricultura Luiz de Queiroz, 1997.

LIMA, J. S. G.; ODINETZ-COLLART, O. Ecologia do camarão Macrobrachium amazonicum (Decapoda, Palaemonidae) no açude Poço da Cruz (Ibimirim). In: CONGRESSO BRASILEIRO DE ENGENHARIA DE PESCA, 10., 1997, Guarapari. Anais... Guarapari: Associação dos Engenheiros de Pesca do Espírito Santo, 1997. p. 378-384.

LIRA, L. P.; SILVA, M. C. N.; CHAVES, R. A. Sobre a Bioecologia do Macrobrachium amazonicum (Heller, 1862) (Crustacea, Decapoda, Palaemonidae) no município de Vigia - Pará - Brasil. In: CONGRESSO BRASILEIRO DE ENGENHARIA DE PESCA, 13. 2003, Porto Seguro. Anais... Porto Seguro: Sociedade Brasileira de Engenharia de Pesca, 2003. p. 59. 
PÉREZ, A. G.; ALSTON, D. E. Comparisons of male and female morphotypes distribution of freshwater prawn, macrobrachium rosenbergii, in monoculture versus polyculture with Nile Tilapia, Oreochromis niloticus. Caribbean Journal of Science, Mayaguez, v. 36, n. 3-4, p. 340-342, 2000.

SANTOS, M. J. M. Policultivo de Tilápia Nilótica (Oreochromis niloticus) e camarão de água doce (Macrobrachium rosenbergii) em sistema semiintensivo de produção. 2001. Dissertação (Mestrado em Aqüicultura) - Centro de Aqüicultura da Universidade de São Paulo, Jaboticabal.

SANTOS, M. J. M.; VALENTI, W. C. Production of Nile tilapia Oreochromisniloticus and freshwater prawn Macrobrachium rosenbergii stocked at different densities in polyculture systems in Brazil. Journal of the World Aquaculture Society, Baton Rouge, v. 33, n. 3, p. 369376, 2002.
TORLONI, C. E. C.; SANTOS, J. J.; CARVALHO JÚNIOR, A. A.; CORRÊA, A. R. A. A pescada-dopiaui Plagioscion squamosissimus (Heckel, 1840) (Osteichthyes, Perciformes) nos reservatórios da Companhia Energética de São Paulo-CESP. São Paulo: CESP/Série Pesquisa e Desenvolvimento, 1993.

UNIVERSIDADE FEDERAL DE VIÇOSA - UFV. SAEG: sistema para análises estatísticas e genéticas: versão 7.1: manual do usuário. Viçosa: Ed. da UFV, 1997.

VALENTI, W. C. Camarão de Água Doce como Agronegócio. In: CONGRESSO DA SOCIEDADE BRASILEIRA DE AQÜICULTURA E BIOLOGIA AQUÁTICA, 1., 2004, Vitória. Anais... Vitória: Aquabio, 2004. p. 52.

ZIMMERMANN, S. Aqüacultura de camarões de água doce: desenvolvimento e perspectivas no Estado do Rio Grande do Sul. Logos, Rio de Janeiro, v. 3, n. 1, p. 55-60, 1991. 
